\title{
Developing a patient information leaflet to improve information offered to patients undergoing appendicectomy
}

Radford Smith, Katie cross

Northern Devon Healthcare NHS Trust, United Kingdom

\begin{abstract}
An appendicectomy is one of the most common operations performed in North Devon District Hospital (NDDH) with over 200 carried out between 2013 to 2014. Despite this, a patient information leaflet (PIL) about appendicectomy is unavailable, which is compromising standards of care and uncompliant with Trust policy.

This project aimed to establish levels of written information offered to patients undergoing an appendicectomy, develop a PIL, and assess its impact on the provision of written information using Plan-Do-Study-Act (PDSA) methodology. Case notes of patients operated on between January 2013 to October 2014 were randomly sampled at baseline. The primary outcome measure was whether written information was offered, retrospectively determined by reviewing the medical record. A PIL was then designed following a standard protocol, published on NDDH's website and distributed onto surgical wards. Posters were displayed in staff rooms to encourage use of the PIL for PDSA cycle 1. An article further promoting the PIL was written for the wards monthly newsletter and a local presentation was delivered for PDSA cycle 2. Patients views about the PIL were assessed prospectively using a questionnaire. The primary outcome was measured at 10 weeks following PDSA cycle 1 and at 5 months following PDSA cycle 2.
\end{abstract}

$17 \%(5 / 30)$ of patients were offered written information at baseline. Following PDSA cycle 1, this rose substantially to $53 \%(8 / 15)$ but rates fell to $46 \%(7 / 15)$ following PDSA cycle $2.89 \%$ of patients (8/9) surveyed agreed or strongly agreed that the PIL was helpful.

This project indicates that provision of written information is poor despite it being highly valued by patients. The first ever Trust appendicectomy PIL has been established which substantially improved provision of written information after 10 weeks. However, continued education of staff is essential to embed good practice over the long term.

\section{Problem}

The consent policy[1] at NDDH in the UK states that written information, including PIL's must be available for patients undergoing procedures requiring written consent, such as an appendicectomy. This is in accordance to GMC guidance[2] that urges clinicians to support their discussions with patients using written material, taking into account varying communication needs, such as patients with hearing impairment. Provision of written information in the pre-operative phase is also recognised by the Royal College of Surgeons of England as a key element in the Enhanced Recovery After Surgery (ERAS) pathway.[3]

Despite this clear guidance, no PIL has been developed for patients undergoing appendicectomy, one of the most common operations carried out in NDDH, with over 200 performed between 2013 to 2014. This issue is compounded by the limited window of opportunity that staff have to provide written information as patients usually present as an emergency.[4] This lack of written material therefore falls short of recognised standards of care and may be compromising patient centred outcomes.

\section{Background}

Although there is limited research on the benefits of PIL's in appendicectomy, there is growing evidence from other surgical specialities suggesting that PIL's are highly valued by patients and may improve patient centred outcomes. In a qualitative study by King \& colleagues,[4] patients perceived that basic information provided by consultant surgeons about the impact of their urgent abdominal surgery on daily activities, including appendicectomy, was lacking and highly variable. To address this issue, procedurespecific written material was created that was found to satisfy patients information needs.

Consistent with this finding, Angioli \& colleagues[5] discovered in a study of 190 patients undergoing surgery for gynaecological malignancy, that those receiving written material pre-operatively were significantly more likely to be satisfied with the information and experience less post-operative pain and subsequent need for analgesia than those given verbal information alone. Pain is a well recognised stimulus of the stress response which has been linked to worse post-operative outcomes. This forms the basis of the Royal College of Surgeons of England's recommendation[3] to provide written information in the pre-operative stage of the Enhanced Recovery After Surgery pathway.

Evidence also suggests that PIL's play a valuable role in facilitating informed consent. In a prospective randomised study, Mauffrey \& 
colleagues[6] analysed 53 patients and demonstrated that those who received PIL's prior to their elective orthopaedic surgery were able to recall significantly more complications than those given routine verbal information.

Despite studies showing the benefits of written material, PIL's are being significantly underused in the healthcare setting. Henney \& colleagues[7] surveyed 50 patients undergoing day-case ENT surgery in a large university teaching hospital and found that written information was offered in just $16 \%$ of cases. A departmental initiative led to an improvement in the number of patients receiving PIL's to $64 \%$, which was achieved by educating staff on the importance of written information through emails and local presentations. In a qualitative study of 100 patients undergoing surgery for colorectal cancer, Litcher \& colleagues[8] found that only $31 \%$ had received written material, despite patients expressing a wish to receive information in a more structured way, including verbal and written information.

\section{Baseline measurement}

The case notes of any patient undergoing appendicectomy between January 2013 to October 2014 were randomly sampled using random number tables generated in excel. The primary outcome measure was whether or not patients were offered written information, determined retrospectively through review of the medical record. Data was also collected on patient age, gender, and whether the operation was laparoscopic, open, diagnostic, or a combination of types. A sample of 30 patients was obtained. Only $17 \%$ (5 out of 30 ) of patients were offered written information. The five patients that received written information were paediatric patients (< 17 years). Three of these patients were given a PIL about pain relief and generic post-operative care. Another two patients were offered a PIL but the details of its contents were not documented. Overall, the rate of paediatric patients receiving written information was $45 \%$ ( 5 out of 11 ). No adult patient was offered written information.

See supplementary file: ds6279.pdf - "Adult appendicectomy PIL"

\section{Design}

A PIL was developed following a standard hospital protocol[9] and comprised of ten sections: What is an appendicectomy, why is it needed, what is involved in removing an appendix, is there an alternative treatment, how will I feel afterwards (including the aftereffects of the anaesthesia, post-operative pain and post-operative nausea and vomiting) what happens after the procedure, what are the risks, aftercare (including medications to take home, resuming daily activities and sport, driving, returning to work and school), and lastly follow up. The content of the PIL was developed in accordance with the literature and in collaboration with senior colorectal surgeons. Adherence to the Clinical Negligence Scheme for Trusts standards and the hospitals Consent to Treatment Policy was maintained.[1,9] It was anticipated that separate adult and paediatric versions of the PIL would be required, as certain information important to adult patients was deemed inappropriate for paediatric patients, such as returning to work and resuming sexual activity. Once the content was finalised, the PIL was submitted to the communications department and readers panel to scrutinize the readability and content of the PIL, a lengthy process taking several weeks. The final version of the PIL was formatted into a standard design for surgical procedures according to the NHS Identity guidelines[10] and published on NDDH's website[11]. This allowed patients and staff to download the material, increasing the accessibility and sustainability of the intervention.

\section{Strategy}

PDSA cycle 1: Copies of the PIL were printed and distributed onto surgical wards and posters highlighting the value of written information and the current poor baseline levels were displayed in doctors and nurses staff rooms. Meetings with ward clerks were held to determine the optimal location on the ward to stock the PIL to make it easily accessible to staff, resulting in the creation of dedicated file cabinet draws. Regular monitoring of patient lists was conducted over the first 10 weeks to assess how frequently the PIL was being offered. Based on feedback from staff, it became evident that the posters were ineffective as nurses and doctors were unaware of the PIL's location or its availability. Other approaches were therefore taken to further promote the PIL and improve its implementation.

PDSA cycle 2: Permission was requested from ward managers to include an article promoting the PIL in the adult ward's monthly newsletter, which was emailed to all nurses and doctors working on relevant wards. A staff meeting was also held for paediatric staff during which the PIL was discussed to encourage its use on the paediatric ward. To further raise its profile and the issues surrounding the current poor levels of written information, a presentation was delivered to the Anaesthetic and Surgical Directorate at their Clinical Governance Day.

A survey was prospectively administered to patients to collect data on their views of the PIL. A Likert scale was used to assess their level of agreement with the following statements: "I found the PIL helpful" and "I would recommend the PIL to a friend". If patients found the PIL helpful, they were invited to share their reasons for this. Overall satisfaction with the PIL was assessed.

\section{Results}

PDSA cycle 1: The case notes of patients of any age undergoing appendicectomy over the first 10 weeks post introduction of the PIL were randomly sampled and the rate of written information offered was determined retrospectively. A sample of 15 patients was obtained. Overall, the percentage of patients who were offered written information rose from $17 \%$ at baseline to $53 \%$ (8 out of 15 ). $75 \%$ ( 3 out of 4 ) of paediatric patients ( $<17$ years) were offered written information.

PDSA cycle 2: The primary outcome was measured again after a 10 week period following implementation of the second cycle. A sample of 15 patients was obtained. This revealed a slight reduction 
in the total number of patients receiving written information from $53 \%$ to $46 \%$ ( 7 out of 15 ). However, the rate of paediatric patients being offered written information rose from $75 \%$ to $83 \%$ (5 out of 6 ).

The survey was prospectively administered to nine patients. $89 \%$ (8 out of 9) either agreed or strongly agreed that they found the PIL helpful. One patient neither agreed nor disagreed that the PIL was helpful. Of those that found the PIL helpful, five patients indicated their reasons for this. For example, one patient stated that it helped "to prepare me for what was ahead including the complications". Another patient stated that the PIL helped to "take away some of the unknown". One patient shared that it was helpful to "refer back to it if I forgot". $67 \%$ ( 6 out of 9 ) of patients strongly agreed that they would recommend the PIL to a friend but one patient neither agreed nor disagreed with this statement. $100 \%$ of patients were satisfied over-all with the PIL.

See supplementary file: ds6581.pdf - "Baseline and postmeasurement table and graph"

\section{Lessons and limitations}

A major challenge of the project was trying to implement the PIL in a setting where offering written information was not routine practice. Methods used such as posters and direct encouragement of staff to offer the PIL was sometimes met by indifference or a lack of motivation, especially on adult wards. This may be because offering written information was seen as a low priority in their clinical duties or an inconvenience.

Furthermore, some staff expressed their concern as to why the focus was on giving PIL's only to those undergoing appendicectomy and not other operations. Developing a Trust led educational programme aimed at fostering a culture of routine offering of PIL's for all operations may therefore be required if a sustainable change in practice is to be achieved. This may involve securing the support of senior medical staff, such as Ward Managers and Consultants who are in a position to influence staff behaviour and promote Trust consent policy, for example in departmental meetings. Collaborating with the Trust's Patient Safety Officer will also be invaluable to exploit their methodologies for embedding change.

However, delivering routine PIL's pre-operatively is a significant challenge for emergency procedures, such as appendicectomy, due to the limited window of opportunity prior to surgery. Therefore, this objective may be more realistic for elective surgery which allows more predictable timing for PIL's, for example during pre-operative assessment clinics.

Rates of paediatric patients receiving written material were consistently higher compared to adults, at baseline and subsequently improved after each PDSA cycle. This may be due to the use of a paper based clinical discharge proforma that prompted nurses to indicate whether a PIL had been given to the patient, which was unavailable on adult wards. However, this usually resulted in patients receiving written material prior to discharge, which precludes its value pre-operatively, such as facilitating informed consent.[6] Therefore, development of a sticker to attach to consent forms reminding clinicians to offer written information preoperatively is suggested as further work to ensure sustainability.

Another issue was that the article included in the wards monthly newsletter promoting the PIL on adult wards would have only been sent to those working on the wards during that time. The high turnover of staff probably made this method ineffective and may in part explain why the rate of patients receiving written information was not sustained after PDSA cycle 2. A more effective approach would have been to create a personalized email for all surgical staff rather than to restrict information to a one-off monthly newsletter. However, the higher proportion of diagnostic laparoscopies in the PDSA cycle 2 sample may have underestimated the true rate of patients receiving the PIL at that stage as patients are unlikely to receive procedure-specific written material if there is diagnostic uncertainty.

The small sample size and information bias from retrospective analysis of notes also limit the conclusions that can be drawn from the results, as the true levels of written information provided may not be accurately reflected in the medical record.

\section{Conclusion}

This project has revealed that substandard levels of written information are currently being offered to patients undergoing appendicectomy in NDDH, despite evidence suggesting that patients highly value this form of information. To address this issue, the first ever Trust adult and paediatric appendicectomy PIL has been successfully established on surgical wards, leading to a substantial improvement in the provision of written material after 10 weeks. However, continuing education of staff is essential to embed a sustainable change in practice that will benefit patients over the long term.

\section{References}

1 James S, Cox B. Consent Policy. Barnstaple: Northern Devon Healthcare NHS Trust; 2014 Aug. 47p. Version 3.3.

http://www.northdevonhealth.nhs.uk/new/wpcontent/uploads/2012/04/Consent-Policy-v3.3-28Aug14.pdf

2 General Medical Council. Consent guidance: Sharing information. http://www.gmc-uk.org/guidance/ethical_guidance/consent guidanc e sharing information.asp

3 Khan S, Gatt M, Horgan A, Anderson I, MacFie J. Issues in Progressional Practice, guidelines for implementation of enhanced recovery protocols. London: Association of Surgeons of Great Britian and Ireland; 2009. http://www.rcseng.ac.uk/healthcarebodies/clinical-policy/standards-and-policy

4 King NK, Cheeseman GA, Siriwardena AK. Development of procedure-specific patient information sheets for acute abdominal surgery and validation in patients undergoing urgent cholecystectomy or appendicectomy. International Journal of Clinical Practice. 2004; 58(6): 559-563. DOI: 
10.1111/j.1368-5031.2004.00017.x (Date accessed 10th October 2015).

5 Angioli R, Plotti F, Capriglione S, et al. The effects of giving patients verbal or written pre-operative information in gynecologic oncology surgery: a randomized study and the medical-legal point of view. European Journal of Obstetrics, Gynaecology and Reproductive Biology. 2014; 177: 67-71. DOI:

10.1016/j.ejogrb.2014.03.041 (Date accessed 15th October 2015).

6 Mauffrey C, Prempeh EM, John J, Vasario G. The influence of written information during the consenting process on patients' recall of operative risks. A prospective randomised study. Internal Orthopaedics. 2008; 32(4): 425-429. DOI:

10.1007/s00264-007-0361-6 (Date accessed 25th October 2015).

7 Henney S, Rakhra S. Patient information in otorhinolaryngology: a prospective audit. Journal of the Royal Society of Medicine Short Reports: 2011; 2(5): 37. DOI: 10.1258/shorts.2011.011022 (Date accessed 20th March 2015)

8 Lithner M, Johansson J, Andersson E, Jakobsson U, Palmquist I, Klefsgard R. Perceived information after surgery for colorectal cancer - an explorative study. Colorectal Disease. 2012; 14(11): 1340-1350. DOI: 10.1111/j.1463-1318.2012.02982.x (Date accessed 5th November 2015).

9 Whitaker I. Producing patient information leaflets procedure. Barnstaple: Northern Devon Healthcare NHS Trust. 2014 Feb. 15p. Version 3.2.

10 NHS Brand Guidelines. Presentation, print and production: general guidance. http://www.nhsidentity.nhs.uk/tools-and-resource s/patient-information/presentation\%2c-print-and-production-generalguidance

11 Smith R. What you should know about your appendicectomy \& what you should know about your child's appendicectomy. Barnstaple: Northern Devon Healthcare NHS Trust; 2015. http://www.northdevonhealth.nhs.uk/category/patient-informationleaflets/surgery/

\section{Declaration of interests}

Nothing to declare

\section{Acknowledgements}

Dr Richard MacMahon, Dr Roope Manhas, John Jarvis

\section{Ethical approval}

The project was exempt from ethical approval as it was deemed a quality improvement project and not a study on human subjects. 